Bio - grafia. Escritos sobre la Biología y su Enseñanza. ISSN 2027

Edición Extraordinaria. p.p. 1113- 1125

Memorias del VIII Encuentro Nacional de Experiencias en Enseñanza de la Biología y la Educación Ambiental. III Congreso Nacional de Investigación en Enseñanza de la Biología.

\title{
UNA PROPUESTA DE EDUCACIÓN AMBIENTAL PARA LA FORMACIÓN CONTINUA DE PROFESORES
}

\section{AN ENVIRONMENTAL EDUCATION PROPOSAL FOR THE CONTINUED TRAINING OF TEACHERS}

B

I

0

Palabras clave: Educación Ambiental; Curso; Educación continua de profesores.

1 Profesor del Departamento de Biología - Universidad Estadual do Norte do Paraná (UENP), Brasil. Correo electrónico: rodrigopoletto@uenp.edu.br;

2 Profesor del Departamento de Biología - Universidad Estadual do Norte do Paraná (UENP), Brasil. Correo electrónico: luckenlucas@uenp.edu.br;

3 Graduada en Negocios Internacionales pela Universidad Santo Tomás de Aquino - Bogotá Colombia. Correo electrónico: 12danieladuarte@gmail.com;

4 Posgrado en Conservación con énfasis en Fragmentación Ambiental pela UENP (Brasil). Correo electrónico: wesleysilva_po@hotmail.com. 
Bio - grafia. Escritos sobre la Biología y su Enseñanza. ISSN 2027

Edición Extraordinaria. p.p. 1113- 1125

Memorias del VIII Encuentro Nacional de Experiencias en Enseñanza de la Biología y la Educación Ambiental. III Congreso Nacional de Investigación en Enseñanza de la Biología.

\begin{abstract}
This article presents the results of an Environmental Education course (EE) performed with seventeen students from an Environmental specialization course at a public university in the state of Paraná (Brazil). All participants were graduates in biological sciences or geography, and worked professionally in teaching. Thus, from a theoretical survey of the subject and the organization of the activities implemented on the course, the activities of the participants were analyzed according to the theoreticalmethodological contributions of discursive textual analysis. As a result, four categories of analysis were obtained with meaning units that evidenced the contributions of the course, according to the opinions of the teachers and the responsibility of each with regard to environmental issues; the role of EE, pollution and polluting agents, and the practices which can be performed to minimize the negative human impact on the environment.
\end{abstract}

Key-words: Environmental Education; Course; Continued training of teachers.

\title{
Introducción
}

El tema de Educación Ambiental (EA) encontró un lugar en el escenario mundial en la década de 1970. Durante este período, una serie de manifestaciones tales como los movimientos feministas, la revolución estudiantil en Francia, los debates políticos en América Latina en medio de gobiernos autoritarios y también, las demandas de una organización democrática que garantizara los derechos humanos básicos, se sumaban a una situación de crisis ambiental evidenciada a mediados del siglo XX, como resultado del crecimiento de las actividades industriales y de la consecuente producción de los residuos y daños al medio ambiente (Medina , 2001).

Así, desde la década de 1970, investigadores de diversos campos han investigado y generado conocimiento en EA para los diferentes niveles educativos, con el fin de capacitar a los adolescentes y jóvenes a llevar una vida con la conciencia y responsabilidad ambiental. En este sentido, en el contexto de la Educación Superior, la educación ambiental se ha presentado cada vez más como un gran desafío frente a las transformaciones socio ambientales constantes e inevitables. Por estas razones, según Leff (2001), la formación ambiental:

es relevante para comprender la transformación de la realidad provocada por el desarrollo. La formación implica un proceso más orgánico y reflexivo de reorganización 
Bio - grafia. Escritos sobre la Biología y su Enseñanza. ISSN 2027

Edición Extraordinaria. p.p. 1113- 1125

Memorias del VIII Encuentro Nacional de Experiencias en Enseñanza de la Biología y la Educación Ambiental. III Congreso Nacional de Investigación en Enseñanza de la Biología.

de los conocimientos y de la sociedad en la construcción de nuevas capacidades de comprender e intervenir en la transformación del mundo. (p. 254)

La universidad y los centros de estudios e investigaciones son, en este sentido, espacios favorables para la construcción de nuevas perspectivas sociales. Siendo así, deben incluir el debate ambiental de forma democrática y amplia. De hecho, desde la conferencia de Tbilisi son presentadas las demandas de formación en EA y desde entonces, nuevas directrices han delineado el programa de EA en todo el sector académico, como las acciones interdisciplinarias para resolver los problemas socio ambientales; el desarrollo de materiales pedagógicos, los programas de formación de profesores, entre otros (Unesco, 1994). Así, según Amaral (2001), desde un punto de vista pedagógico y animado por este crecimiento, la EA se ha consolidado como un aspecto innovador del acto educativo siendo ampliamente considerado, a fin de abordar las temáticas ambientales en todos los sectores la sociedad como un tema interdisciplinar y transversal.

Del mismo modo, para Reigota (2004), la formación de ciudadanos conscientes, activos y participativos sería el objetivo de mayor importancia para el presente siglo por la complejidad de los problemas socio ambientales existentes. Por lo tanto, frente a las necesidades de desarrollo de propuestas educativas orientadas a la formación de profesores en EA, pero con una perspectiva de acción local, se presenta a continuación, las etapas de un curso realizado con estudiantes de una especialización en Medio Ambiente de una Universidad Pública de Paraná (Brasil), así como los resultados de este proyecto pedagógico a través de un análisis de los relatos de los participantes. Los datos generales de este curso son:

Cuadro 01 - Datos del Curso

\begin{tabular}{|l|l|}
\hline \multicolumn{2}{|c|}{ CURSO DE FORMACIÓN DOCENTE EM EDUCACIÓN AMBIENTAL } \\
\hline Contexto & $\begin{array}{l}\text { El curso fue realizado en } 2014 \text { teniendo como público objetivo a los graduados } \\
\text { de pregrados en geografía y ciencias biológicas. En total fueron diecisiete } \\
\text { participantes. }\end{array}$ \\
\hline Objetivo & Promover la formación pedagógica en EA. \\
\hline Carga horaria & Tres reuniones con ocho horas de duración. \\
\hline Actividades & $\begin{array}{l}\text { Dentro y fuera de clase, envolviendo estudio de textos, investigaciones, trabajo } \\
\text { de campo, dinámicas y actividades. }\end{array}$ \\
\hline
\end{tabular}


Bio - grafia. Escritos sobre la Biología y su Enseñanza. ISSN 2027

Edición Extraordinaria. p.p. 1113- 1125

Memorias del VIII Encuentro Nacional de Experiencias en Enseñanza de la Biología y la Educación Ambiental. III Congreso Nacional de Investigación en Enseñanza de la Biología.

\begin{tabular}{|l|l|}
\hline $\begin{array}{l}\text { Conocimientos } \\
\text { previos }\end{array}$ & Nociones de ecología general, química, física y ciencias. \\
\hline Valoración & En todas las cátedras, por medio de actividades teóricas y prácticas. \\
\hline Recursos & $\begin{array}{l}\text { Televisión (videos e imágenes), tablero de tiza, materiales reciclables, papel, } \\
\text { cinta adhesiva, tijeras, títeres, visitas técnicas, artículos científicos y libros } \\
\text { didácticos. }\end{array}$ \\
\hline
\end{tabular}

Fuente: De los autores

En síntesis, las actividades desarrolladas en los tres días del curso fueron:

$1^{\circ}$ Cátedra: el curso comenzó con la recepción de los estudiantes y la aplicación de una evaluación diagnóstica inicial sobre la temática ambiental. Posteriormente, se inició la clase con una dinámica llamada Árbol de los Sueños, por medio de la cual, los estudiantes intentaban definir en una sola palabra lo que esperaban del curso y en seguida estas palabras eran escritas en el tablero. Cada palabra componía una parte de un esquema ramificado en forma de árbol, donde cada hoja contenía una palabra presentada por los participantes y todas estaban vinculadas a un tronco principal, con raíces. De este modo, se demostró que todas las ideas de los estudiantes serían abordadas durante las cátedras, siendo el tronco del árbol el objetivo común del curso: la formación en EA de manera integral y vivencial. Subsiguientemente, se abordó el tema del agua, mostrando su papel en el equilibrio y la sustentabilidad de la vida, así como la información del ciclo hidrológico y sobre cómo los humanos usan inapropiadamente este recurso natural. También se habló de la importancia de manantiales, bosques y vegetación ribereña. Se mostró en el mapamundi el Acuífero Guaraní - uno de los mayores reservorios de agua dulce en el mundo y que abarca varios estados de Brasil y otros países de América Latina - proporcionando información general sobre el mismo. En la misma cátedra se realizó una dinámica conocida como Observación del Ambiente, en la que los estudiantes debían salir del aula, elegir una ubicación para observar $y$, desde la punta de los pies hasta donde la vista alcanzara en el horizonte, harían una observación detallada de todo, realizando una evaluación crítica respecto al equilibrio del ambiente visualizado. Estos registros fueron recogidos, leídos y discutidos en grupos. También se realizó una discusión de los temas: calidad del aire, vegetación, ritmo urbano, deforestación y sostenibilidad. El día terminó con una visita técnica a una plaza donde los estudiantes analizaron las condiciones ambientales, haciendo uso de las nociones trabajadas anteriormente en el curso. Por último, recibieron la tarea de tabular los residuos producidos en sus hogares de residencia, investigar el destino de 
Bio - grafia. Escritos sobre la Biología y su Enseñanza. ISSN 2027

Edición Extraordinaria. p.p. 1113- 1125

Memorias del VIII Encuentro Nacional de Experiencias en Enseñanza de la Biología y la Educación Ambiental. III Congreso Nacional de Investigación en Enseñanza de la Biología.

estos residuos, el origen del agua utilizada en sus hogares, y hacer un registro fotográfico de un problema ambiental urbano.

$2^{\circ}$ Cátedra: Se inició con una charla sobre ecología, fauna y desastres ambientales. Luego se trató sobre la temática Residuos, en especial sobre residuos sólidos urbanos. Se analizó la tabulación de los datos recogidos de la investigación que se había proporcionado a los estudiantes como trabajo durante la cátedra anterior y en seguida se realizó una lectura sobre la relación consumo/globalización, la cual motivó una amplia discusión acerca del uso indiscriminado de envases de plástico. Se abordó también, temáticas como el secuestro de carbono en el planeta, la producción de combustibles no fósiles, el suelo y sus variedades, alteraciones, la contaminación, la recuperación y su papel en la agricultura. Más tarde hubo una dinámica de observación de basuras en los alrededores de la Universidad, seguida por la presentación de un video relacionado con este tema, como retroalimentación. Se realizó otro ejercicio en grupo, que consistió en compartir el conocimiento de cada uno sobre los "problemas ambientales" de las ciudades de la cual provenía cada estudiante. Se les solicitó que relataran los problemas detectados, sus causas y soluciones, estimulándolos a realizar una análisis crítico de las condiciones de sus barrios, ciudades y del lugar visitado durante el curso. Este análisis fue guiado por un cuestionario. En este día, también se recibió la visita de agentes ambientales (municipales), quienes presentaron un video sobre la forma en que tratan los residuos sólidos en la ciudad. La última actividad del segundo día del curso consistió en la división por grupos de los estudiantes, a fin de realizar una lectura de la Ley Nacional (brasileña) de EA y la elaboración de un Proyecto Ambiental a ser desarrollado en la región.

$3^{\circ}$ Cátedra: Se discutió sobre la fauna brasileña, con énfasis en la fauna regional, y se llegó a los conceptos de cadena y red alimentaria. En seguida, se promovió una reflexión sobre las principales causas de la degradación de la vida silvestre, tales como la caza furtiva, los incendios, la destrucción de hábitats naturales y el atropellamiento de los animales silvestres. Se incluyó en la discusión la fauna doméstica y los cuidados y maltratos de estos animales. Como actividad, se desarrollaron dinámicas como: Fruta en el árbol, Cadena Alimentaria y Nudo Ciego, las cuales trataron sobre temas de "equilibrio ecológico" y "biodiversidad". Se realizó la plantación de árboles frutales en el perímetro de la Universidad. En esta práctica, hubo una formación previa del profesorado acerca de las dimensiones, preparación de los agujeros y cuidados con las mudas. Posteriormente, se realizó otra visita técnica a la plaza anteriormente visitada, donde se desarrolló un trabajo 
Bio - grafia. Escritos sobre la Biología y su Enseñanza. ISSN 2027

Edición Extraordinaria. p.p. 1113- 1125

Memorias del VIII Encuentro Nacional de Experiencias en Enseñanza de la Biología y la Educación Ambiental. III Congreso Nacional de Investigación en Enseñanza de la Biología.

de concienciación de los habitantes de la región. Esta actividad funcionó como una retroalimentación de los temas trabajados en las cátedras anteriores. Los participantes del curso fueron capaces de comentar sobre la experiencia de atender a las personas y hablar de EA desde una mirada orientada a posibles acciones de conservación. Por último, todos

hicieron una evaluación diagnóstica final del curso. El trabajo fue finalizado con una merienda colectiva.

De esta manera, después de presentar las actividades realizadas en el transcurso del curso, a continuación se delinearán los procedimientos metodológicos adoptados en el análisis de las evaluaciones de los participantes del curso.

\section{Metodología}

El presente estudio, de ámbito cualitativo, implicó la realización de una revisión y recopilación bibliográfica (Bogdan y Biklen, 1994) acerca de la EA, así como también el análisis de la producción escrita de los estudiantes a través de un análisis textual discursivo. En total, fueron diecisiete participantes egresados de pregrados en ciencias biológicas o geografía, todos con una experiencia en docencia en Educación Básica Brasileña, que osciló entre seis meses y tres años. De acuerdo con los supuestos teórico metodológicos de Moraes y Galiazzi (2006), las respuestas de las evaluaciones aplicadas a los participantes del curso constituyeron el corpus de análisis, siendo sometidas a las etapas previstas en este referencial, que son: "la unitarización - deconstrucción de los textos del corpus; la categorización - establecimiento de vínculos entre los elementos unitarios; y por último, la captura de un nuevo emergente en el que la nueva comprensión es comunicada y validada" ( $p$ 192). Las respuestas analizadas en este artículo están relacionadas con cuatro preguntas relativas a los instrumentos de evaluación mencionados anteriormente, de la siguiente manera: A) Para usted, ¿qué es la educación ambiental?; B) En su opinión, ¿cuáles son los objetivos de la educación ambiental?; C) iUsted genera "contaminación" en el día a día? En caso afirmativo, explique.; D) ¿Usted realizó o realiza algún tipo de acción que considera pertenece a la perspectiva de la educación ambiental?. A continuación, se presenta un análisis de las respuestas a estas preguntas a través de un análisis textual discursivo de las respuestas de los estudiantes.

\section{Resultados y Discusión}


Bio - grafia. Escritos sobre la Biología y su Enseñanza. ISSN 2027

Edición Extraordinaria. p.p. 1113- 1125

Memorias del VIII Encuentro Nacional de Experiencias en Enseñanza de la Biología y la Educación Ambiental. III Congreso Nacional de Investigación en Enseñanza de la Biología.

Para garantizar el anonimato de los estudiantes del curso, los cuales autorizaron la utilización de sus respuestas en el presente estudio, los mismos fueron codificados de la siguiente forma: se utilizó la letra " $C$ " acompañada de algoritmos ordinales (C1, C2... C17) para referirse a los participantes del curso. La segunda codificación se refiere al día de la cátedra en el que la actividad escrita fue realizada, siendo E1 para el primer día de cátedra y E2 para el tercero y último día de cátedra.

De esta manera, siguiendo las etapas subsecuentes del análisis textual discursivo, anteriormente mencionadas, fue posible configurar cuatro categorías y diferentes unidades de análisis/sentido para las mismas. Para ejemplificar este proceso de categorización y análisis de datos, se presentan en las Cuadros 02, 03, 04 e 05 los segmentos textuales de algunos estudiantes que resultan representativos de los demás participantes:

Cuadro 02 - Categoría "Nociones de Educación Ambiental"

Descripción: Esta categoría reúne fragmentos de las transcripciones de los estudiantes, que evidencian las nociones que los mismos presentaron antes del inicio del curso, respecto a la Educación Ambiental.

\begin{tabular}{|c|c|}
\hline & $N$ \\
\hline $\begin{array}{l}\text { Unidades de } \\
\text { entido/análisis: } \\
\text { Perspectiva } \\
\text { social }\end{array}$ & $\begin{array}{l}\text { No recuerdo. C11, E1. } \\
\text { Es saber respetar los espacios naturales de manera general. C15, E1. } \\
\text { Es una ciencia que estudia la naturaleza, sus componentes y medios para } \\
\text { conservar estos elementos. C17, E1. }\end{array}$ \\
\hline $\begin{array}{l}\text { Unidades de } \\
\text { sentido/análisis: } \\
\text { Perspectiva } \\
\text { personal }\end{array}$ & $\begin{array}{l}\text { Es todo lo que envuelve el medio en que vivimos, desde el respeto entre las } \\
\text { personas y a la naturaleza hasta la reducción personal de residuos que cada } \\
\text { uno genera por su propio consumo, para que cada uno de nosotros pueda } \\
\text { contribuir con la permanencia de la vida. C4, E2. } \\
\text { Comprende la importancia de una grande área de conocimiento que ayuda a las } \\
\text { personas a entender lo que es realmente el medio ambiente (lo que nos rodea) } \\
\text { y a darnos la base para que podamos hacer nuestra parte, en el día a día y } \\
\text { ayudar a la permanencia de la naturaleza viva. C11, E2. }\end{array}$ \\
\hline
\end{tabular}


Bio - grafia. Escritos sobre la Biología y su Enseñanza. ISSN 2027

Edición Extraordinaria. p.p. 1113- 1125

Memorias del VIII Encuentro Nacional de Experiencias en Enseñanza de la Biología y la Educación Ambiental. III Congreso Nacional de Investigación en Enseñanza de la Biología.

Es tener conciencia y respetar el medio ambiente. Es tener conocimiento de cómo vivir en armonía con el medio ambiente, pero cada uno debe hacer su parte, comenzando por sí mismo. C15, E2.

Fuente: De los autores

Cuadro 03 - Categoría "Objetivos de la Educación Ambiental"

Descripción: Esta categoría reúne fragmentos textuales que expresan la comprensión de los estudiantes con respecto a los objetivos de la Educación Ambiental, con énfasis en su relevancia y consolidación como área de conocimiento y campo formativo.

Unidades de No sabría decir. C1, E1.

sentido/análisis:

Desconocimiento No sé C3,E1.

\begin{tabular}{|c|c|}
\hline $\begin{array}{l}\text { Unidades de } \\
\text { entido/análisis: } \\
\text { Nociones } \\
\text { incompletas }\end{array}$ & $\begin{array}{l}\text { Comprende el mejorar la convivencia de las personas en general con el medio } \\
\text { ambiente y trabajar en la educación de jóvenes y adultos. C9, E1. } \\
\text { Hacer que las personas comprendan el significado de medio ambiente y su } \\
\text { entorno. C16, E1. }\end{array}$ \\
\hline $\begin{array}{l}\text { Unidades de } \\
\text { sentido/análisis: } \\
\text { Nociones } \\
\text { próximas a la } \\
\text { comprensión } \\
\text { científica actual }\end{array}$ & $\begin{array}{l}\text { Formar personas críticas para que practiquen el uso racional de los recursos } \\
\text { naturales y ayudar en la construcción de la sociedad de modo sustentable. C1, } \\
\text { E2. } \\
\text { El objetivo de la educación ambiental es concienciar a las personas en el } \\
\text { presente para que en el futuro las generaciones que vendrán encuentren } \\
\text { posibilidades suficientes de vida con respecto al uso de la naturaleza. } \\
\text { Preservar para durar más para las generaciones futuras. C3, E2. } \\
\text { Es concienciar la población, orientándola a la práctica de un desarrollo } \\
\text { sustentable que debe comenzar en pequeños gestos diarios.C9, E2. } \\
\text { Es una meta de la educación ambiental el trabajar para educar a las personas } \\
\text { de diferentes niveles de educación para tener un contacto más racional con } \\
\text { el medio, generando menos impactos negativos en la naturaleza y pensando en } \\
\text { maneras para recuperar áreas degradadas y preservar áreas nativas, uniendo } \\
\text { esto con el desarrollo social. C16, E2. }\end{array}$ \\
\hline
\end{tabular}


Bio - grafia. Escritos sobre la Biología y su Enseñanza. ISSN 2027

Edición Extraordinaria. p.p. 1113- 1125

Memorias del VIII Encuentro Nacional de Experiencias en Enseñanza de la Biología y la Educación Ambiental. III Congreso Nacional de Investigación en Enseñanza de la Biología.

Fuente: De los autores

Cuadro 04 - Categoría "Contaminación"

Descripción: abarca segmentos de las respuestas de los participantes del curso, evidenciando la precepción de los mismos con respecto a la contaminación que cada uno genera en sus actividades personales.

\begin{tabular}{|c|c|}
\hline $\begin{array}{l}\text { Unidades de } \\
\text { sentido/análisis: } \\
\text { Adjudicación } \\
\text { impersonal de } \\
\text { responsabilidad }\end{array}$ & $\begin{array}{l}\text { Todas las personas generan grandes cantidades de basura diariamente, no } \\
\text { hay manera de evitar esto. C2, E1. } \\
\text { Si. Toda la población afecta de alguna manera el planeta contaminándolo de } \\
\text { manera inevitable. C5, E1. } \\
\text { Claro que si. Desde su nacimiento el ser humano genera contaminación. C13, } \\
\text { E1. }\end{array}$ \\
\hline $\begin{array}{l}\text { Unidades de } \\
\text { sentido/análisis: } \\
\text { Adjudicación } \\
\text { personal de } \\
\text { responsabilidad }\end{array}$ & $\begin{array}{l}\text { Yo contamino en todo momento, cuando utilizo el carro, cuando compro y uso } \\
\text { productos envasados uno dentro de otro. C2, E2. } \\
\text { Yo ciertamente contamino. Inclusive cuando me cepillo los dientes estoy } \\
\text { desechando el envase de la crema de dientes, contaminando el agua y asi } \\
\text { sucesivamente en las diferentes actividades. C5, E2. } \\
\text { Desde las ropas que utilizo hasta los envases de los productos que uso. } \\
\text { Siempre dejo "residuos" en el ambiente. C13, E2. }\end{array}$ \\
\hline
\end{tabular}

Fuente: De los autores

Cuadro 05 - Categoría "Acciones de Educación Ambiental"

Descripción: En esta categoría están incluidos los segmentos textuales de las evaluaciones de los participantes del curos referentes a las posibles acciones ambientales que estuvieran realizando.

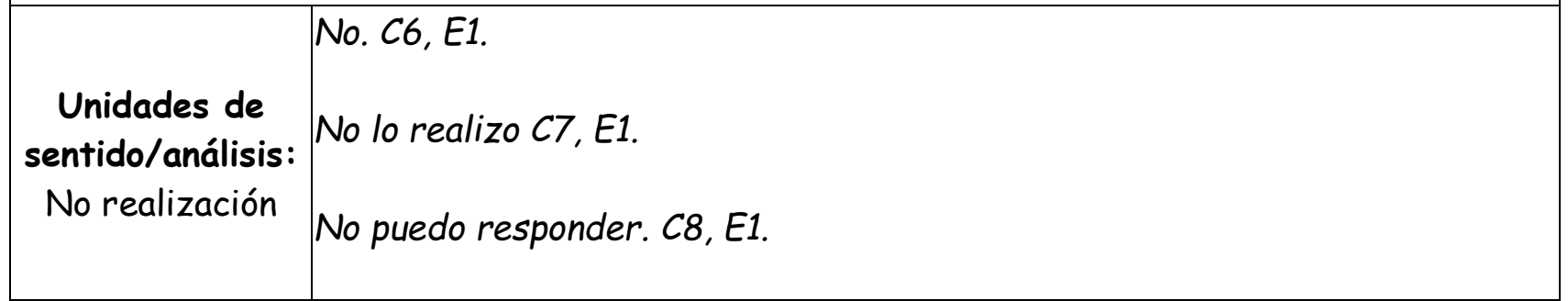


Bio - grafia. Escritos sobre la Biología y su Enseñanza. ISSN 2027

Edición Extraordinaria. p.p. 1113- 1125

Memorias del VIII Encuentro Nacional de Experiencias en Enseñanza de la Biología y la Educación Ambiental. III Congreso Nacional de Investigación en Enseñanza de la Biología.

\begin{tabular}{|c|c|}
\hline & $\begin{array}{l}\text { Creo que no. C10, E1. } \\
\text { En la práctica no. C12, E1. } \\
\text { Nunca desarrollé acciones en el medio ambiente. C14, E1. }\end{array}$ \\
\hline $\begin{array}{c}\text { Unidades de } \\
\text { sentido/análisis: } \\
\text { Realización } \\
\text { después de la } \\
\text { participación en el } \\
\text { curso }\end{array}$ & $\begin{array}{l}\text { Sí. En el presente curso comencé a no usar vasos plásticos en cada cátedra, } \\
\text { ahora traigo mi propio vaso y aunque es poco es un comienzo. C6, E2. } \\
\text { Sí, disminuyendo la producción de residuos, caminando en vez de utilizar el } \\
\text { carro para ir al trabajo. C7, E2. } \\
\text { Sí, ahora procuro siempre no arrojar basura en lugares inadecuados. C8, E2. } \\
\text { Sí, ya estoy acostumbrado a ahorrar algunos recursos como el agua. C10, E2 } \\
\text { Sí, separo los reciclables en mi casa y apago las luces cuando no hay nadie en } \\
\text { el lugar. C12, E2. } \\
\text { De los proyectos no, pero practico acciones en el día a día, como plantar }\end{array}$ \\
\hline
\end{tabular}

Fuente: De los autores

A partir de los datos fue posible evidenciar en la categoría 1 "Nociones de Educación Ambiental" que los estudiantes antes de iniciar el curso propuesto, presentaron nociones de EA orientadas a la impersonalidad, como si la responsabilidad de cada uno frente a las temática ambiental fuera dispersa en el ámbito social, como visto en C4E1, C11E1, C15E1 y C17E1. Ahora, al final del periodo formativo los mismos participantes presentaron explicaciones direccionadas a la responsabilidad personal citando, inclusive, la necesidad de acciones diarias y personales conforme fue mencionado en C4E2, C11E2, C15E2 y C17E2.

En la Categoría 2 "Objetivos de la Educación Ambiental" fue notable, a partir de las unidades de sentido / contexto, que en la evaluación diagnóstica inicial los participantes del curso no sabían destacar los objetivos de la EA (C1E1 y C3E3) o tenían nociones, por ejemplo, argumentando que es objetivo de la EA [...]mejorar la convivencia de las personas en general con el medio ambiente y trabajar en la educación de jóvenes y adultos (C9, E1) o 
Bio - grafia. Escritos sobre la Biología y su Enseñanza. ISSN 2027

Edición Extraordinaria. p.p. 1113- 1125

Memorias del VIII Encuentro Nacional de Experiencias en Enseñanza de la Biología y la Educación Ambiental. III Congreso Nacional de Investigación en Enseñanza de la Biología.

bien, Hacer que las personas comprendan el significado de medio ambiente y su entorno (C16, E1). En ambos casos, no se aborda el papel de la EA en la formación para el uso racional de los recursos naturales orientado hacia el desarrollo sostenible de las economías - la formación crítica de personas que practiquen acciones ambientalmente responsables. No obstante, no se presentaron, los objetivos específicos que explicitaran el papel de EA como un área de investigación y campo de conocimiento. Sin embargo, estos se percibieron en la evaluación diagnóstica final de los participantes (C1E2, C3E2, C9E2 y C16E2), en quienes se evidenció, las contribuciones del curso en la clarificación y el suministro de información teórico práctica para una correcta comprensión de los objetivos de EA. Esta categoría 2, por lo tanto hacer recordar las palabras de Reigota (2004), en lo que concierne a las contribuciones de la formación de ciudadanos conscientes, actuantes y participativos mediante la complejidad de los problemas sociales y ambientales existentes. Se percibe que el curso permitió, como lo evidencia el autor anteriormente citado, que los participantes después de recibir la formación significativa y eficiente, demostraran una mayor comprensión de las cuestiones ambientales, presentando respuestas con definiciones más próximas de las comprensiones científicas actuales sobre EA, mostrando así, la relevancia de la formación proporcionada.

Continuando con la Categoría 3 "Contaminación", así como con las categorías anteriores, los participantes del curso mostraron a partir de sus respuestas, que antes del curso no tenían una percepción clara de cuánto cada persona contamina el medio ambiente todos los días. Las respuestas siempre tenían una perspectiva colectiva (C2E1, C5E1 y C13E1) y la misma tendencia se observó en la primera categoría. Al final del curso, sin embargo, se pudo observar que la contaminación pasó a ser vista desde una perspectiva personal, a partir de diferentes ejemplificaciones: Yo contamino en todo momento, cuando utilizo el carro, cuando compro y uso productos envasados uno dentro de otro (C2, E2), o igualmente Siempre dejo "residuos" en el ambiente (C13, E2).

La cuarta y última categoría "Acciones de Educación Ambiental" algunos participantes registraron en la evaluación diagnóstica inicial, que nunca habían realizado o realizaban acciones de perspectiva ambiental (C6E1, C7E1, C8E1, C10E1, C12E1 y C14E1). No obstante, este patrón fue alterado en la evaluación final, de modo que los participantes comenzaron a presentar acciones concretas, gracias a la influencia del curso, según argumentaron: C6E2, C7E2, C8E2, C10E2, C12E2 y C14E2. Pertinentemente, Leff (2001, p. 254) presenta las contribuciones de la formación en EA como una formación que "[...] implica un proceso más 
Bio - grafia. Escritos sobre la Biología y su Enseñanza. ISSN 2027

Edición Extraordinaria. p.p. 1113- 1125

Memorias del VIII Encuentro Nacional de Experiencias en Enseñanza de la Biología y la Educación Ambiental. III Congreso Nacional de Investigación en Enseñanza de la Biología.

orgánico y reflexivo de reorganización del conocimiento de la sociedad en la construcción de nuevas capacidades para comprender e intervenir en la transformación del mundo". Este proceso de construcción de nuevas capacidades fue percatado en la fase final del curso, cuando los participantes evidenciaron, como visto en esta categoría, intervenciones prácticas en sus realidades personales.

\section{Consideraciones Finales}

En este artículo se muestran los resultados de un curso de formación docente en Educación Ambiental para profesores de las áreas de ciencias biológicas y geografía. Después de delimitar el esquema teórico que fundamentó la construcción de la propuesta (curso en EA), se presentó la estructura de la misma a partir de las principales actividades realizadas.

Mediante un análisis textual discursivo de las evaluaciones realizadas por los participantes del curso antes y después del curso, se notó que la intervención pedagógica trajo contribuciones a los participantes, entre las cuales es posible citar: una comprensión más adecuada de la EA según el reconocimiento de responsabilidades no solamente colectivas sino también individuales (categoría 1); una visión más clara del rol de la EA en la formación de las personas desde los años iniciales del ciclo básico de enseñanza hasta la integración de los mismos a la vida en sociedad, según la vivencia de principios económicos que respeten el medio ambiente (Categoría 2); una comprensión más adecuada de los tipos de contaminantes y de los agentes contaminadores (categoría 3); y por último, una reflexión personal propia en cuanto a las acciones que cada uno puede practicar (y muchos relataron haber hecho esto a partir del curso) con el fin de contribuir para el establecimiento de una cultura de cuidado y respeto del medio ambiente (categoría 4).

De esta forma, a partir de las categorías obtenidas con el análisis de los datos, se concluyó que el curso trajo contribuciones a los participantes que mostraron, entre otras cosas, una mejoría en la comprensión de los asuntos abordados en una postura más clara frente a sus acciones diarias y la perspectiva ambiental. Con estos resultados, se destaca la relevancia de propuestas formativas en EA, con objetivos didácticos y metodológicos adecuadamente definidos, con la finalidad de contribuir en la formación continua de agentes multiplicadores de buenas prácticas, en diferentes áreas del conocimiento. 
Bio - grafia. Escritos sobre la Biología y su Enseñanza. ISSN 2027

Edición Extraordinaria. p.p. 1113- 1125

Memorias del VIII Encuentro Nacional de Experiencias en Enseñanza de la Biología y la Educación Ambiental. III Congreso Nacional de Investigación en Enseñanza de la Biología.

\section{Referencias}

Amaral, I. A. (2001). Educação Ambiental e ensino de Ciências: uma história de controvérsias. Pro-Posições. Campinas, v. 12, n. 1 (34).

Bogdan, R., \& Biklen, S. (1994). Investigação qualitativa em educação: uma introdução à teoria e aos métodos. Portugal: Porto.

Leff, E. (2001). Saber Ambiental. Petrópolis-RJ: Vozes.

Medina, N. M. (2001). A formação dos professores em educação fundamental. In: Vianna, L.P. (Coord.). Panorama da Educação Ambiental no Ensino Fundamental. Brasília: MEC, SEF.

Moraes, R., \& Galiazzi, M. C. (2006). Análise textual discursiva: processo reconstrutivo de múltiplas faces. Ciência \& Educação, Bauru, SP, v. 12, n. 1, p. 117-128. Disponível em: $\langle\mathrm{http}: / /$ www2.fc.unesp.br/cienciaeeducacao/include/getdoc. php?id=801\&article=154\& mode=pdf $>$. Acesso em: 16 fev. 2015.

Reigota, M. O. (2004). O que é Educação ambiental. Coleção Primeiros Passos. São Paulo: Brasiliense.

Unesco. (1994). Programa Internacional de Educación Ambiental. Estratégias para la formación del profesorado en educación ambiental. Vasco: CENEAN, libros de la Catarara, n. 25. 